\title{
Autophagy Inhibition Contributes to ROS-Producing NLRP3-Dependent Inflammasome Activation and Cytokine Secretion in High Glucose-Induced Macrophages
}

\author{
Jiezhi Daia Xiaotian Zhang ${ }^{\mathrm{a}} \quad$ Li Li $^{\mathrm{b}}$ Hua Chen ${ }^{\mathrm{a}}$ Yimin Chai ${ }^{\mathrm{a}}$ \\ aDepartment of Orthopedic Surgery, Shanghai Sixth People's Hospital, JiaoTong University, Shanghai,

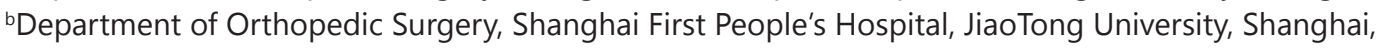 \\ China
}

\section{Key Words}

Macrophages $•$ NLRP3 inflammasome $\bullet$ IL-1 1 Autophagy $\bullet$ ROS

\begin{abstract}
Background: Type 2 diabetes is a persistent inflammatory response that impairs the healing process. We hypothesized that stimulation with high glucose following a pro-inflammatory signal would lead to autophagy inhibition, reactive oxygen species (ROS) production and eventually to the activation of the Nod-like receptor protein (NLRP) -3. Methods: Macrophages were isolated from human diabetic wound. We measured the expression of NLRP3, caspase1 and interleukin- 1 beta (IL-1 $\beta$ ) by western blot and real-time PCR, and the surface markers on cells by flow cytometry. THP-1-derived macrophages exposed to high glucose were applied to study the link between autophagy, ROS and NLRP3 activation. LC3-II, P62, NLRP3 inflammation and IL-1 $\beta$ expression were measured by western blot and real-time PCR. ROS production was measured with a Cellular Reactive Oxygen Species Detection Assay Kit. Results: Macrophages isolated from diabetic wounds exhibited a pro-inflammatory phenotype, including sustained NLRP3 inflammasome activity associated with IL-1ß secretion. Our data showed that high glucose inhibited autophagy, induced ROS production, and activated NLRP3 inflammasome and cytokine secretion in THP-1-derived macrophages. To study high glucose-induced NLRP3 inflammasome signalling, we performed studies using an autophagy inducer, a ROS inhibitor and a NLRP3 inhibitor and found that all reduced the NLRP3 inflammasome activation and cytokine secretion. Conclusion: Sustained NLRP3 inflammasome activity in wound-derived macrophages contributes to the hyper-inflammation in human diabetic wounds. Autophagy inhibition and ROS generation play an essential role in high glucose-induced NLRP3 inflammasome activation and cytokine secretion in macrophages.
\end{abstract}

Hua Chen,

and Yimin Chai

Department of Orthopedic Surgery, Shanghai Sixth People's Hospital,

JiaoTong University, Shanghai (China)

Tel. +8618930174887, Fax 008602164701361, E-Mail chuadr@aliyun.com/ymchai@sjtu.edu.cn

\section{KARGER}




\section{Cellular Physiology Cell Physiol Biochem 2017;43:247-256 \begin{tabular}{ll|l} 
and Biochemistry & $\begin{array}{l}\text { DOI: 10.1159/000480367 } \\
\text { Published online: August 30, } 2017\end{array}$ & $\begin{array}{l}\text { (c) } 2017 \text { The Author(s). Published by S. Karger AG, Basel } \\
\text { www.karger.com/cpb }\end{array}$
\end{tabular} \\ Dai et al:: Autophagy, ROS and NLRP3 Inflammasome}

\section{Introduction}

An impaired wound healing process characterizes diabetes and may result in the adverse events in type 2 diabetic patients [1]. A persistent inflammatory response is exhibited, with the extended accumulation of macrophages and increased levels of pro-inflammatory cytokines $[2,3]$. Macrophages play an important role in each phase of wound healing. Metabolic changes cause a macrophage disorder, which may be expected to impair healing in diabetes. Studies have shown that excessive amounts of glucose have direct effects on the polarization of primary human macrophages in vitro towards an M1-like phenotype [4], characterized by the up-regulation of the cluster of differentiation (CD) 11c or CC chemokine receptor (CCR7) as well as the down-regulation of CD206 or arginase1 (Arg-1) [5, 6]. In our study, the M1/M2 imbalance in macrophages isolated from human diabetic wounds is also assessed.

Recently, much interest has evolved concerning the role of the Nod-like receptor protein (NLRP) -3 inflammasome which is a molecular platform for immune defence. It is a multiprotein complex that consists of the adaptor molecule apoptosis-associated speck-like protein containing a caspase recruitment domain (ASC) and the effector enzyme caspase1 $[7,8]$. Activation of the NLRP3 inflammasome results in the production of interleukin-1 beta (IL-1 $\beta$ ) and interleukin-18 (IL-18) [9]. Because inflammation plays a prominent role in chronic organ injury, the activation of the NLRP3 inflammasome and the increased proinflammatory cytokines have been implicated in the pathogenesis of numerous chronic diseases, including diabetes [10].

It has been recently shown that autophagy can regulate the inflammasome, and it probably inhibits the intracellular signalling by removing dysfunctional mitochondria that would otherwise increase the production of intracellular reactive oxygen species (ROS) [11]. Excess of ROS can cause cellular dysfunction and inflammation. A recent study suggests that ROS may lead to the formation of the NLRP3 inflammasome and subsequently activate the secretion of IL-1 $\beta$ and IL-18 [12].

An in vivo study has shown that activation of the NLRP3 inflammasome is one of the key contributors to the delayed healing of wounds in diabetic mice [13]. To further access the inflammatory response mechanism in diabetic wounds, we hypothesized that stimulation with high glucose following a pro-inflammatory signal would lead to autophagy inhibition, reactive oxygen species (ROS) production and eventually to activation of the Nod-like receptor protein (NLRP) -3. Our results therefore provide a possible mechanism underlying inflammation in impaired diabetic wound healing. THP-1-derived macrophages are used in this study.

\section{Materials and Methods}

\section{Human subjects}

We included six type 2 diabetes patients in this study who had chronic wounds located anywhere on the foot that had lasted for at least three months. We collected wound tissue located near the centre of the wound during initial debridement as part of the standard of care. Non-diabetic wound tissues harvested from discarded tissues of patients undergoing traumatic amputation were used as a control. This study was approved by the Ethics Review Board of Shanghai Six People's Hospital affiliated to Shanghai Jiao Tong University. Written informed consent was obtained from all the enrolled participants.

\section{Cell culture and treatment}

Most studies were carried out in the human monocytic cell line THP-1, which was obtained from the Cell Bank of the Chinese Academy of Sciences (Shanghai, China) and maintained in RPMI 1640 media supplemented with $10 \% \mathrm{FBS}$ at $37{ }^{\circ} \mathrm{C}$ with $5 \% \mathrm{CO}_{2}$. To induce cell differentiation, the cells were seeded in macrophage serum-free medium with Phorbol-12-myristate-13-acetate (PMA, Sigma-Aldrich, Darmstadt, Germany) for $72 \mathrm{~h}$. For stimulation, cells were incubated with $30 \mathrm{mM}$ glucose (HG) for $48 \mathrm{~h}$. To exclude a 


\section{Cellular Physiology Cell Physiol Biochem 2017;43:247-256 \begin{tabular}{l|l|l} 
and Biochemistry & DOI: 10.1159/000480367 30,2017 & $\begin{array}{l}\text { C) } 2017 \text { The Author(s). Published by S. Karger AG, Basel } \\
\text { www.karger.com/cpb }\end{array}$ \\
\hline
\end{tabular} \\ Dai et al.: Autophagy, ROS and NLRP3 Inflammasome}

hyperosmolar effect, we added $30 \mathrm{mM}$ mannitol (M) as an osmotic pressure control. Moreover, cells were treated with DMSO, resveratrol $(50 \mu \mathrm{M}), \mathrm{NAC}(5 \mathrm{mM})$ and BAY 11-7082 $(20 \mu \mathrm{M})$ for $4 \mathrm{~h}$ at $37^{\circ} \mathrm{C}$ respectively.

\section{Cell isolation}

Cell isolation was done according to the method described by Mriza et al [14].. Cells were dissociated from human chronic wound biopsies using an enzymatic digest with collagenase I, collagenase XI and hyaluronidase. Neutrophils, T cells and B cells were depleted from the total cell population with CD15, CD3 and CD 19 antibodies, and magnetic sorting. Cells of the monocyte/Mp lineage were then isolated using CD11b magnetic beads.

Western blot

Antibodies directed at caspase1, ASC, IL-1 $\beta$, P62 and LC3-II were purchased from Santa Cruz (CA, USA); and antibodies directed at NLRP3 were purchased from Abcam (Cambridge, UK).

RNA Analysis

RNA was reverse-transcribed to cDNA and used to quantify the amount of NLRP3, caspase-1, ASC and IL-1 $\beta$ mRNA by real-time PCR. Relative gene expression was determined using the $2^{-\Delta \triangle C T}$ method and $\beta$-actin was used as an endogenous control.

Measurement of ROS production

Cellular ROS generation was measured with a Cellular Reactive Oxygen Species Detection Assay Kit (Red Fluorescence), which was purchased from Abcam (Cambridge, UK). The cells were prepared in growth medium and ROS Red Dye Working Solution was added. After incubating at $37^{\circ} \mathrm{C}$ for 60 minutes, the cells were treated with test compounds to induce them. Finally, the fluorescence intensity increase was monitored at an excitation wavelength of $520 \mathrm{~nm}$ and an emission wavelength of $605 \mathrm{~nm}$ with the bottom read mode.

\section{Characterization of cell surface markers by flow cytometry}

After collecting the macrophages, $5 \times 10^{5}$ cells were re-suspended in $50 \mu \mathrm{L}$ of sterile PBS. Then, the cells were simultaneously incubated with $2 \mu \mathrm{g}$ of anti-CCR7 or $6 \mu \mathrm{g}$ of anti-206 for $45 \mathrm{~min}$ on ice. After incubation and three rounds of washing in the same buffer, the cells were incubated with FITC anti-mouse antibody for 25 min on ice. The samples were analysed using BD FACSCalibur and the results were analysed using the FlowJo software.

\section{Data analysis}

Data were expressed as the mean \pm SD and processed using the SPSS 18.0 statistical software (SPSS Inc., Chicago, USA). Student's t-test was used to compare two groups. A one-way analysis of variance (ANOVA) was used to assess the differences between multiple groups, followed by Tukey's post hoc test. A P-value < 0.05 was considered significant. All statistical analyses were performed using the GraphPad Prism software.

\section{Results}

Expression of NLRP3, caspase1 and IL-1ß in human diabetic wound isolated macrophages

We compared the level of NLRP3 inflammasome and IL-1 $\beta$ gene and protein expression in macrophages from diabetic or non-diabetic wounds. A significantly higher level of NLRP3, ASC, caspase 1 and IL-1 $\beta$ mRNA expression is found in macrophages isolated from diabetic wounds compared to non-diabetic wounds $(\mathrm{P}<0.05)$ (Fig. $1 \mathrm{~A}-\mathrm{D})$. Consistent with the mRNA expression pattern, a similar expression profile of the protein expression of NLRP3, ASC, caspase 1 and IL-1 $\beta$ is observed in the diabetic wound group compared with the non-diabetic group (Fig. $1 \mathrm{E}-\mathrm{I}$ ).

Expression of surface markers in human diabetic wound isolated macrophages

M1 macrophages usually express a high level of cell surface markers such as CCR7, while M2 macrophages express a higher level of the mannose receptor (CD206) [15]. We accessed 
Fig. 1. mRNA and protein expression of NLRP3, caspase1 and IL-1 $\beta$ in macrophages isolated from diabetic wound (MP/D) and non-diabetic wound (MP). RNA are measured by real-time PCR (A-D) and proteins are measured by Western blot (E-I), respectively. Values are mean \pm SD; $* \mathrm{p}<0.05$.

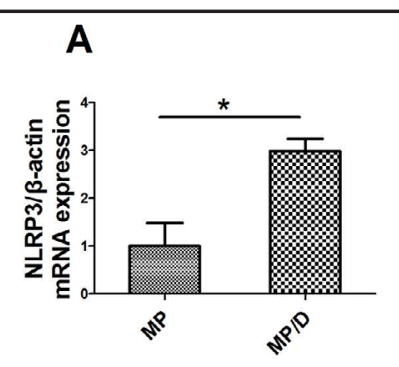

B

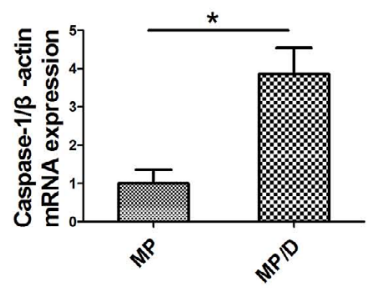

E

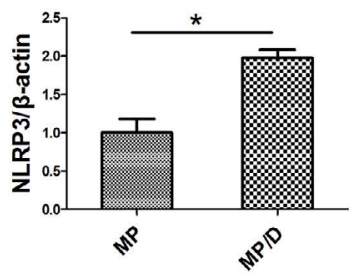

H

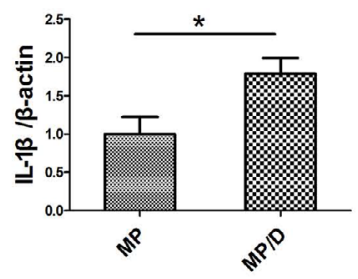

C

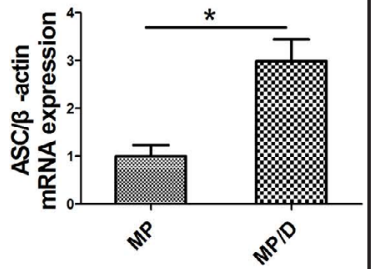

$\mathbf{F}$

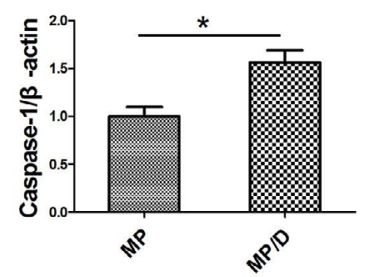

I

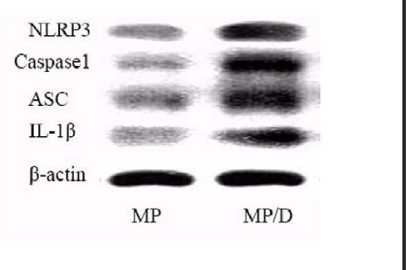

Fig. 2. Cell-surface CCR7 (A, C, E) and CD206 (B, D, F) expression in macrophages isolated from diabetic wound (MP/D) and non-diabetic wound (MP). Values are mean \pm SD; * $\mathrm{p}<0.05$.

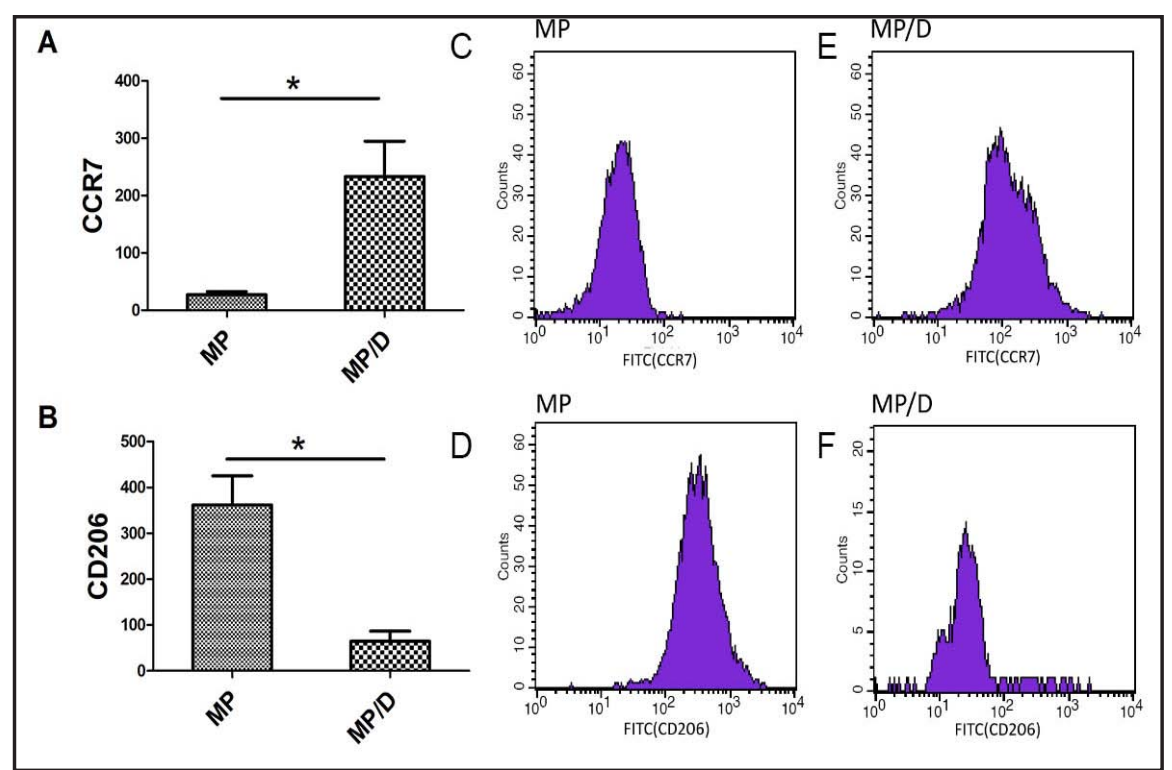

the expression of these surface markers by flow cytometry. As expected, the expression of CCR7 is significantly up-regulated on macrophages isolated from diabetic wound, with the decreased expression of CD206 (Fig. 2 A, B). 
Fig. 3. High glucose induces expression of the NLRP3 inflammasome, ROS generation and autophagy in THP-1-derived macrophages. The protein level of LC3-II and P62 are measured using Western blot analysis (A, F, G). The intracellular ROS production is measured with fluorometry (H). The mRNA and protein levels of NLRP3, ASC, Caspase1 and IL-1 $\beta$ are measured by realtime PCR (I-L) and Western blot (A-E), respectively. Values are mean \pm $\mathrm{SD} ;{ }^{*} \mathrm{p}<0.05$.

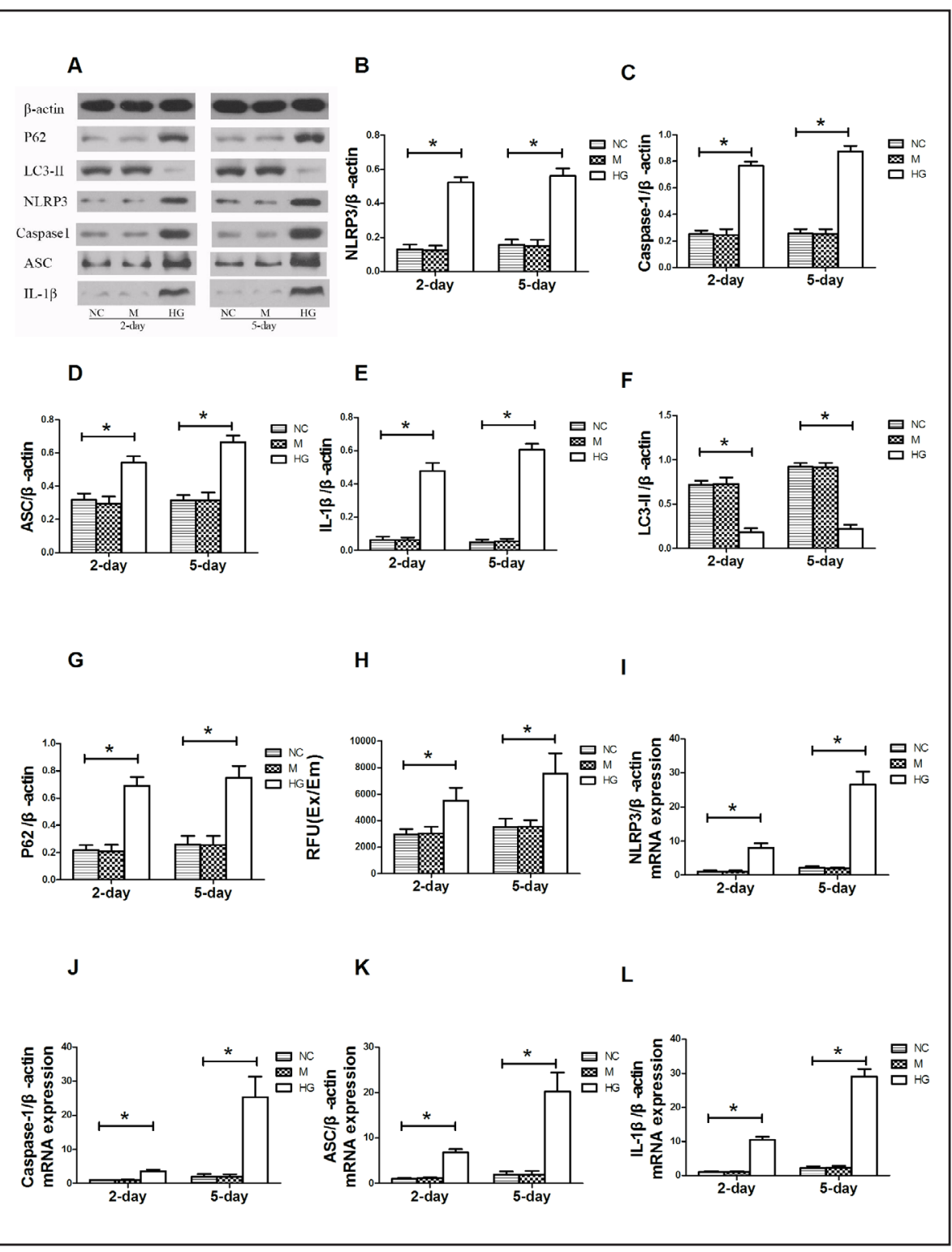

High glucose inhibits autophagy and induces ROS production, NLRP3 inflammasome activation and cytokine secretion

To determine the effect of high glucose on autophagy, ROS production and NLRP3 inflammasome activation, THP-1-derived macrophages are incubated in a medium containing high glucose (30 mM) for two days or five days. To exclude a hyperosmolar effect, we added $30 \mathrm{mM}$ mannitol as an osmotic pressure control. As expected, mannitol had no effect on the protein and gene expression.

LC3-II actively participates in autophagosome formation and is a well-accepted hallmark of autophagy induction [16]. The level of P62, another autophagy marker, is regulated by the balance between its transcriptional regulation (incoming flux) and the post-translation autophagic degradation (outgoing flux) [17]. LC3-II is dramatically reduced at the protein level in high glucose-treated cells compared to the control, while P62 is significantly increased, indicating a suppressed autophagy flux (Fig. 3 A, F, G).

We used the ROS-sensitive cellular ROS detection assay kit to access whether a high glucose medium induces ROS production from cultured macrophages. A significant increase of ROS production is observed in the high glucose group compared with the control at both 
A

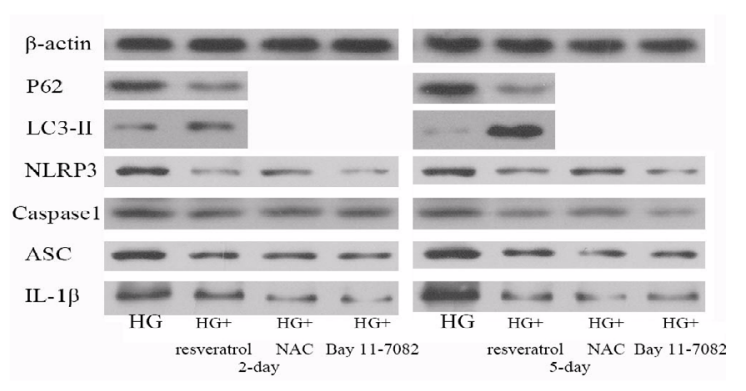

C

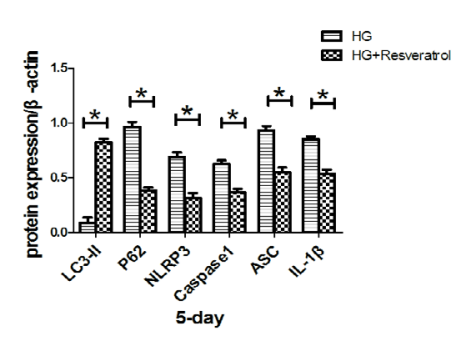

$\mathbf{F}$

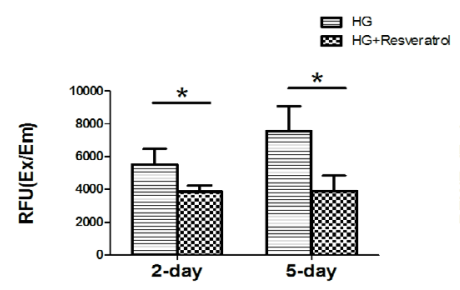

I

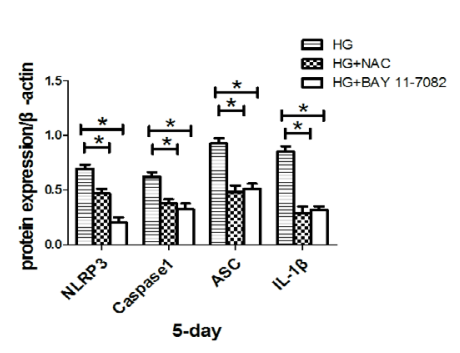

D

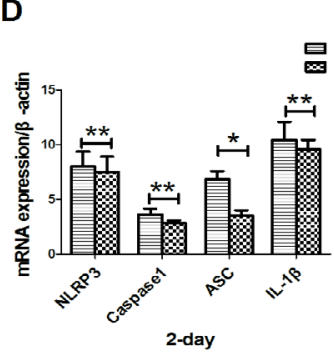

G

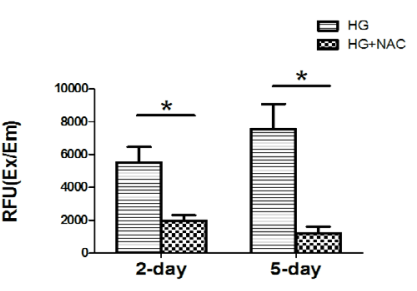

J
B

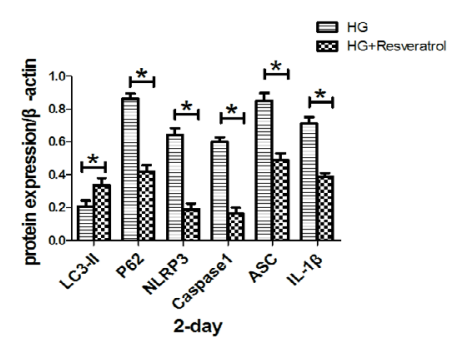

E

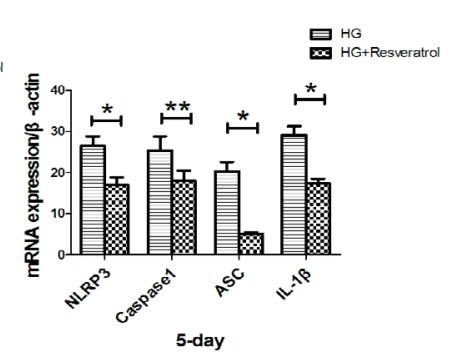

H

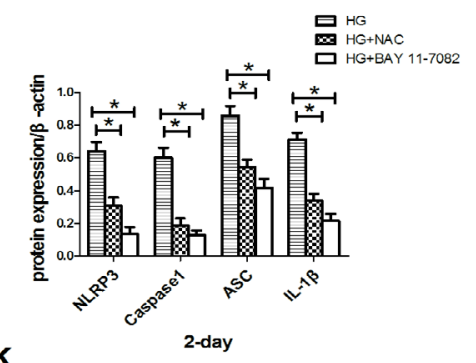

K

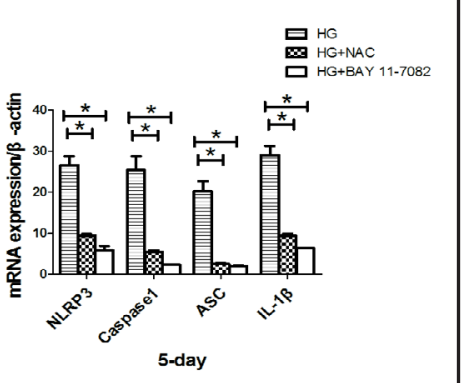

Fig. 4. The role of resveratrol, NAC and BAY 11-7082 treatment on the expression of autophagy, ROS generation and the NLRP3 inflammasome activation in high glucose-induced THP-1-derived macrophages. Resveratrol treatment induces autophagy (A-C), and reduces ROS generation (F) and the NLRP3 inflammasome activation (A-E). NAC decreases ROS generation (G) and the NLRP3 inflammasome activation (A, H-K). BAY 11-7082 reduces both the NLRP3 inflammasome and cytokine expression in high glucoseinduced macrophages $(\mathrm{A}, \mathrm{H}-\mathrm{K})$. Values are mean $\pm \mathrm{SD}$; ${ }^{*} \mathrm{p}<0.05$; ** $\mathrm{p}>0.05$.

two and five days ( $\mathrm{p}<0.05$ ) (Fig. $3 \mathrm{H}$ ). Compared with the control, NLRP3, caspase1, ASC and IL-1 $\beta$ are significantly induced at both the mRNA and protein levels following two and five days of exposure to $30 \mathrm{mM}$ glucose $(\mathrm{p}<0.05)$, suggesting that a high glucose concentration increased the NLRP3 inflammasome levels in a time-dependent manner (Fig. 3 A-E, I-L).

\section{KARGER}




\section{Cellular Physiology Cell Physiol Biochem 2017;43:247-256

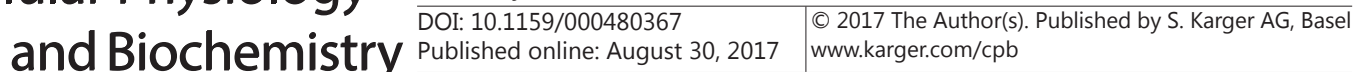

Role of autophagy in the high glucose-promoted activation of the NLRP3 inflammasome

An intimate connection exists between autophagy and ROS. We speculate that the enhancement of autophagy would reduce the accumulation of ROS-producing damaged mitochondria, which would then inhibit the activation of the NLRP3 inflammasome. To determine the role of autophagy involved in high glucose-induced NLRP3 activation and the related pathway in THP-1-derived macrophages, the cells are treated with resveratrol, an autophagy agonist. As expected, resveratrol enhanced LC3-II expression (Fig. 4 A-C). Pretreatment with resveratrol results in a marked decrease in cellular ROS production and the protein expression of the NLRP3 inflammasome (Fig. 4 A-C, F). The protein level of the pro-inflammatory cytokine IL-1 $\beta$ is decreased following the down-regulation of the NLRP3 inflammasome (Fig. 4 A-E). However, the mRNA expression of NLRP3, caspase-1 and IL$1 \beta$ is not significantly decreased at two days (Fig. $4 \mathrm{D}$ ). These results suggest that the ROS production and NLRP3 inflammasome activation participate in an autophagy-associated inflammatory response.

Involvement of ROS production in high glucose-induced NLRP3 inflammasome activation

ROS scavenger NAC application markedly decreases ROS production in macrophages compared with the control (Fig. 4 G). Similarly, NLRP3, ASC, caspase 1 and IL-1 $\beta$ transcriptional levels are down-regulated following ROS inhibition. These changes indicate that ROS inhibition suppresses NLRP3 inflammasome activation (Fig. 4 A, H-K).

The NLRP3 inflammasome blockade and activation of downstream pathways in high glucose

High glucose induces an increased and persistent activation of the NLRP3 inflammasome. An NLRP3 blockade by BAY 11-7082 is confirmed by the protein and mRNA expression in cultured macrophages at two and five days. As a direct consequence of the NLRP3 blockade, a reduction of ASC and caspase 1 activation is observed, at two and five days. Decreased levels of the cytokine IL- $1 \beta$ in cultured macrophages are sustained at two and five days, compared with the control. A NLRP3 blockade by BAY 11-7082 markedly reduces both the NLRP3 inflammasome and cytokine expression in high glucose-induced macrophages at each time point (Fig. 4 A, H-K).

\section{Discussion}

Chronic diabetic wounds are typically associated with a persistent inflammatory response that involves the accumulation of macrophages. Previous evidence has reported the NLRP3 inflammasome acts as a key regulatory pathway in the chronic inflammatory response in macrophages and diabetic wounds [18]; however, little is known about the regulation and function of the NLRP3 inflammasome in macrophages. NLRP3 is unique in its ability to recognize molecular patterns associated with host-derived signals that are abundant in obese individuals, including excess ATP, glucose, ROS, etc [19]. Mitophagy is a highly conserved cellular pathway designed to degrade damaged mitochondria and decrease ROS generation during times of metabolic stress. Therefore, we wanted to clarify the link between ROS generation, autophagy and the NLRP3 inflammasome activation in macrophages. Towards this goal, we employ THP-1- derived macrophages and treat these cells with high glucose to minic an inflammatory state. Our findings show that an autophagy deficiency contributes to ROS-producing NLRP3-dependent inflammasome activation and cytokine secretion in high glucose-primed macrophages.

IL-1 $\beta$ acts as 'master' pro-inflammatory cytokine mediator that plays a role in the pathophysiology of many inflammatory diseases, including diabetes. It participates in a proinflammatory positive feedback loop that sustains the pro-inflammatory macrophage (M1) phenotype in poorly healing wounds [3]. During impaired healing of diabetic wounds, it is characterized by the prolonged accumulation of pro-inflammatory macrophage phenotype 


\section{Cellular Physiology Cell Physiol Biochem 2017;43:247-256 \\ \begin{tabular}{ll|l} 
DOI: 10.1159/000480367 & Ond 2017 The Author(s). Published by S. Karger AG, Basel \\
www.karger.com/cpb
\end{tabular} \\ Dai et al.: Autophagy, ROS and NLRP3 Inflammasome}

associated with increased levels of pro-inflammatory cytokines, such as TNF- $\alpha$, IL-6, and IL-8, and decreased levels of various growth factors, such as TGF- $\beta$, VEGF and EGF. Data from our study and other studies support a role for the wound environment in regulating the macrophage phenotype and, in particular, sustaining a pro-inflammatory phenotype in diabetic wound macrophages. In our study, the pro-inflammatory macrophage phenotype up-regulates in macrophages isolated from human diabetic wounds, and in other studies, macrophages undergo M1-type pro-inflammatory polarization from high levels of glucose exposure in in vitro culture conditions [6].

Increased IL-1 $\beta$ expression can cause widespread tissue damage, which can be found in diabetes and diabetic wounds. The production of IL-1 $\beta$ is critically regulated by the NLRP3 inflammasome [20]. High levels of the NLRP3 inflammasome and IL-1 $\beta$ gene and protein expression are found in macrophages isolated from human diabetic wounds. Our data also demonstrates an increase in the NLRP3 inflammasome and IL-1 $\beta$ gene and protein expression in THP-1-derived macrophages from high glucose exposure, while NLRP3 downregulation by BAY 11-7082 blocks this effect.

ROS arise from the accumulation of damaged, ROS-generating mitochondria, which are caused by a mitophagy/autophagy blockade [21]. Environmental stresses such as high glucose, lipid peroxidation and protein oxidation can dramatically increase ROS production, resulting in significant cell damage. Zhou et al. [11] found that increased ROS production can trigger the NLRP3 inflammasome. Mitochondrial damage characterized by robust mitochondrial ROS generation and loss of membrane potential is linked to the NLRP3 inflammasome activation, and manipulations that block any of these processes inhibit inflammasome activation [22]. Our in vitro data demonstrates that ROS generation from high glucose exposure up-regulate the NLRP3 inflammasome activation and IL-1 $\beta$ protein and gene expression, and the ROS inhibitor NAC markedly down-regulates this effect. These results suggest that the NLRP3 inflammasome mediates ROS-induced IL- $1 \beta$ secretion in macrophages. As autophagy leads ROS-generating mitochondria clearance constantly, it is logically supposed that direct modulation of autophagy wounds contributes to interference with NLRP3 inflammasome activation.

Autophagy selectively removes unneeded organelles such as mitochondria by a process known as mitophagy [23]. It is an important part of immune cell function and shapes subsequent immune responses and is also important for mitochondrial quality control. Poor quality mitochondria may enhance cellular oxidative stress, generate apoptosis signals, and induce cell death [24]. In the context of inflammation and the immune response, the roles of autophagy seem to be diverse - it occurs as an adaptive or protective repose to environmental alterations. Ma et al [25]. reported that autophagy was enhanced in podocytes cultured under high glucose conditions. However, in the present study, we show that high glucose inhibits autophagic activity, as evidenced by a decrease in LC3-II expression and the accumulation of the P62 protein. This result is consistent with Xin et al's [26] study, in which high glucose was demonstrated to inhibit autophagy in podocytes, and autophagy was activated by a rapamycin-ameliorated cell injury. It is hypothesized that this discrepancy between the studies might be due to the difference in the time for which the cells were exposed to high glucose. In the study by Ma, autophagy was analysed at $24 \mathrm{~h}$, while in our study, autophagy is analysed at two and five days. Wang et al [27]. reported that the expression of LC3-II was increased and the expression of P62 was decreased in a high glucose $(11.0 \mathrm{mM})$ group. When the glucose concentration was $22.0 \mathrm{mM}$, P62 expression was increased and the LC3-II protein was degraded, which suggests that the inhibition of autophagy under high glucose in macrophages is a beneficial adaptive response that protects cells against high glucose toxicity. Therefore, the level of autophagy might be affected by the duration and the concentration of high glucose stimulation.

To access a direct role of autophagy in the inflammation process, macrophages are treated with the autophagy agonist resveratrol. Reduced ROS concentrations and the NLRP3 inflammasome activation are confirmed following the induction of autophagy and 


\section{Cellular Physiology Cell Physiol Biochem 2017;43:247-256

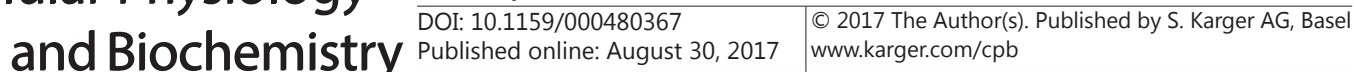 \\ Dai et al.: Autophagy, ROS and NLRP3 Inflammasome}

a subsequent decline of IL- $1 \beta$ secretion in the human THP-1 macrophage cell line. It was also demonstrated by a previous study that the inhibition of mitophagy with 3-MA led to the accumulation of ROS-producing mitochondrial damage and, as a consequence, to the activation of the NLRP3 inflammasome in ARPE-19 cells [28]. On the other hand, the mRNA expression of NLRP3, caspase-1 and IL-1 $\beta$ are not decreased significantly at two days. It seemed that autophagy would affect the NLRP3 inflammasome activation rather than mRNA expression in the early phase.

\section{Conclusion}

In conclusion, our study demonstrates that in THP-1-derived macrophages, a high glucose-induced autophagy deficiency increases ROS generation, which can trigger the NLRP3 inflammasome, whose subsequent activation leads to IL-1 $\beta$ secretion. The link between autophagy, ROS generation and activation of the NLRP3 inflammasome in macrophages suggests a novel pathway underlying inflammation in impaired diabetic wound healing. These data may provide a basis for the further development of a potential therapeutic strategy for improving healing in diabetic patients.

\section{Acknowledgements}

This study was approved by the Ethics Review Board of Shanghai Six People's Hospital affiliated to Shanghai Jiaotong University

Sponsorship for this study and article processing charges was by Foundation of Shanghai Sixth People's Hospital (ynlc201505).

\section{Disclosure Statement}

All the authors declare that they have no Disclosure Statement.

\section{References}

1 Falanga V: Wound healing and its impairment in the diabetic foot. Lancet 2005;366:1736-1743.

-2 Mast BA, Schultz GS: Interactions of cytokines, growth factors, and proteases in acute and chronic wounds. Wound Repair \& Regeneration 1996;4:411-420.

3 Mirza RE, Fang MM, Ennis WJ, Koh TJ: Blocking interleukin-1beta induces a healing-associated wound macrophage phenotype and improves healing in type 2 diabetes. Diabetes 2013;62:2579-2587.

- Liu W, Zhang X, Zhao M, Chi J, Liu Y, Lin F, Fu Y, Ma D, Yin X: Activation in M1 but not M2 Macrophages Contributes to Cardiac Remodeling after Myocardial Infarction in Rats: a Critical Role of the Calcium Sensing Receptor/NRLP3 Inflammasome. Cell Physiol Biochem 2015;35:2483-2500.

5 Zhang X, Dai J, Li L, Chen H, Chai Y: NLRP3 Inflammasome Expression and Signaling in Human Diabetic Wounds and in High Glucose Induced Macrophages. Journal of Diabetes Research 2017;2017:5281358.

-6 Torres-Castro I, Arroyo-Camarena UD, Martinez-Reyes CP, Gomez-Arauz AY, Duenas-Andrade Y, HernandezRuiz J, Bejar YL, Zaga-Clavellina V, Morales-Montor J, Terrazas LI, Kzhyshkowska J, Escobedo G: Human monocytes and macrophages undergo M1-type inflammatory polarization in response to high levels of glucose. Immunol Lett 2016;176:81-89.

7 Martinon F, Burns K, Tschopp J: The inflammasome: a molecular platform triggering activation of inflammatory caspases and processing of proIL-beta. Mol Cell 2002;10:417-426.

-8 Huang Z, Zhuang X, Xie C, Hu X, Dong X, Guo Y, Li S, Liao X: Exogenous Hydrogen Sulfide Attenuates High Glucose-Induced Cardiotoxicity by Inhibiting NLRP3 Inflammasome Activation by Suppressing TLR4/NFkappaB Pathway in H9c2 Cells. Cell Physiol Biochem 2016;40:1578-1590. 


\section{Cellular Physiology Cell Physiol Biochem 2017;43:247-256

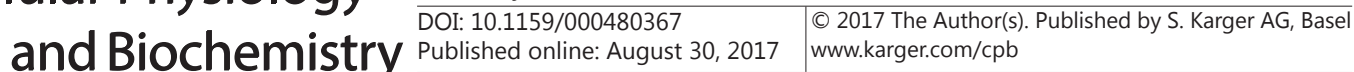

Dai et al.: Autophagy, ROS and NLRP3 Inflammasome

-9 Haasken S, Sutterwala FS: Damage control: management of cellular stress by the NLRP3 inflammasome. Eur J Immunol 2013;43:2003-2005.

10 Bracey NA, Gershkovich B, Chun J, Vilaysane A, Meijndert HC, Wright JR, Jr., Fedak PW, Beck PL, Muruve DA, Duff HJ: Mitochondrial NLRP3 protein induces reactive oxygen species to promote Smad protein signaling and fibrosis independent from the inflammasome. J Biol Chem 2014;289:19571-19584.

11 Zhou R, Yazdi AS, Menu P, Tschopp J: A role for mitochondria in NLRP3 inflammasome activation. Nature 2011;469:221-225.

$\checkmark 12$ Lee HM, Kim JJ, Kim HJ, Shong M, Ku BJ, Jo EK: Upregulated NLRP3 inflammasome activation in patients with type 2 diabetes. Diabetes 2013;62:194-204.

13 Bitto A, Altavilla D, Pizzino G, Irrera N, Pallio G, Colonna MR, Squadrito F: Inhibition of inflammasome activation improves the impaired pattern of healing in genetically diabetic mice. Br J Pharmacol 2014;171:2300-2307.

14 Mirza R, Koh TJ: Dysregulation of monocyte/macrophage phenotype in wounds of diabetic mice. Cytokine 2011;56:256-264.

15 Labonte AC, Tosello-Trampont AC, Hahn YS: The role of macrophage polarization in infectious and inflammatory diseases. Moleculer Cells 2014;37:275-285.

16 Ding Z, Wang X, Schnackenberg L, Khaidakov M, Liu S, Singla S, Dai Y, Mehta JL: Regulation of autophagy and apoptosis in response to ox-LDL in vascular smooth muscle cells, and the modulatory effects of the microRNA hsa-let-7 g. Int J Cardiol 2013;168:1378-1385.

17 Puissant A, Fenouille N, Auberger P: When autophagy meets cancer through p62/SQSTM1. American Journal of Cancer Research 2012;2:397-413.

18 Mirza RE, Fang MM, Weinheimer-Haus EM, Ennis WJ, Koh TJ: Sustained inflammasome activity in macrophages impairs wound healing in type 2 diabetic humans and mice. Diabetes 2014;63:1103-1114.

19 Mori MA, Bezy 0, Kahn CR: Metabolic syndrome: is Nlrp3 inflammasome a trigger or a target of insulin resistance? Circulation Research 2011;108:1160-1162.

-20 Abderrazak A, Syrovets T, Couchie D, El Hadri K, Friguet B, Simmet T, Rouis M: NLRP3 inflammasome: from a danger signal sensor to a regulatory node of oxidative stress and inflammatory diseases. Redox Biol 2015;4:296-307.

21 Wu J, Li X, Zhu G, Zhang Y, He M, Zhang J: The role of Resveratrol-induced mitophagy/autophagy in peritoneal mesothelial cells inflammatory injury via NLRP3 inflammasome activation triggered by mitochondrial ROS. Exp Cell Res 2016;341:42-53.

22 Yang S, Xia C, Li S, Du L, Zhang L, Zhou R: Defective mitophagy driven by dysregulation of rheb and KIF5B contributes to mitochondrial reactive oxygen species (ROS)-induced nod-like receptor 3 (NLRP3) dependent proinflammatory response and aggravates lipotoxicity. Redox Biol 2014;3:63-71.

23 Ding Z, Liu S, Wang X, Dai Y, Khaidakov M, Deng X, Fan Y, Xiang D, Mehta JL: LOX-1, mtDNA damage, and NLRP3 inflammasome activation in macrophages: implications in atherogenesis. Cardiovasc Res 2014;103:619-628.

24 Ashrafi G, Schwarz TL: The pathways of mitophagy for quality control and clearance of mitochondria. Cell Death Differ 2013;20:31-42.

25 Ma T, Zhu J, Chen X, Zha D, Singhal PC, Ding G: High glucose induces autophagy in podocytes. Exp Cell Res 2013;319:779-789.

26 Xin W, Li Z, Xu Y, Yu Y, Zhou Q, Chen L, Wan Q: Autophagy protects human podocytes from high glucoseinduced injury by preventing insulin resistance. Metabolism 2016;65:1307-1315.

27 Wang X, Feng Z, Li J, Chen L, Tang W: High glucose induces autophagy of MC3T3-E1 cells via ROS-AKTmTOR axis. Mol Cell Endocrinol 2016;429:62-72.

-28 Shi H, Zhang Z, Wang X, Li R, Hou W, Bi W, Zhang X: Inhibition of autophagy induces IL-1beta release from ARPE-19 cells via ROS mediated NLRP3 inflammasome activation under high glucose stress. Biochem Biophys Res Commun 2015;463:1071-1076. 